\title{
Physics Case and Challenges for the Vertex Tracker at Future High Energy $e^{+} e^{-}$Linear Colliders
}

\author{
M. Battaglia \\ CERN, CH-1211 Geneva 23, Switzerland
}

\begin{abstract}
The physics programme of high energy $e^{+} e^{-}$linear colliders relies on the accurate identification of fermions in order to study in details the profile of the Higgs boson, search for new particles and probe the multi-TeV mass region by means of precise electro-weak measurements and direct searches.
\end{abstract}

Key words: vertex detector; linear collider

\section{Introduction}

With the end of the LEP program and the start of operation of the $B$-factories, the next step in experimentation at lepton colliders will be at linear accelerators (LC) able to deliver $e^{+} e^{-}$collisions at centre-of-mass energies $\sqrt{s}$ in the range $0.3-1.0 \mathrm{TeV}$ with a luminosity of the order of $10^{34} \mathrm{~cm}^{-2} \mathrm{~s}^{-1}$ and eventually to achieve $\sqrt{s}=3-5 \mathrm{TeV}$ with luminosity of $10^{35} \mathrm{~cm}^{-2} \mathrm{~s}^{-1}$ or higher. The lower end of this energy range will be devoted to the accurate study of the Higgs profile, if a relatively light Higgs boson exists as suggested by the LEP and SLC data, to the precise determination of the top mass and to complement the LHC program in searching for signals of new physics beyond the Standard Model. A multi-TeV linear collider is expected to break new grounds, by exploring the mass scale beyond $10 \mathrm{TeV}$ for new phenomena and to study in detail the properties of the new physics, established by the LHC or the first LC phase.

Present projects focus on three different beam acceleration techniques: warm RF (mostly X-band as for the NLC (1) and JLC (2) projects) or superconducting cavities (such as TESLA (3)) and two-beam acceleration at high frequency (such as CLIC (田)). The proposed technologies being optimised for different $\sqrt{s}$ energies, the full energy range can be covered by subsequent upgrades using the same infrastructures or by planning two distinct phases corresponding to different accelerators. 
The Vertex Tracker of the LC detector is expected to provide the LC data with the fermion flavour tagging capabilities that are instrumental to the understanding of the electro-weak symmetry breaking mechanism and in the search for new particles. Several Vertex Tracker designs have been proposed, relying on different sensor technologies. A common denominator of these proposals is the use of pixel devices due to the high particle density and the emphasis on the material budget minimisation to improve the track extrapolation performances at small momenta in multi-jet final states.

\section{Experimental Conditions for the Vertex Tracker}

The Vertex Tracker at the linear collider will be exposed to background and radiation levels and to track densities unprecedented for $e^{+} e^{-}$collider machines. The main source of background in the interaction region is due to $e^{+} e^{-}$pairs produced and bent in the intense electro-magnetic interaction of the colliding beams. The hit densities have been estimated for each collider design using dedicated beam simulation programs and tracking of the produced particles in the detector solenoidal field. The radius and maximum length of the innermost sensitive layer are defined by the envelope of the deflected particles from pairs. Results are summarised in Table 1.

Table 1

Pair hit density on innermost Vertex Tracker layer for different LC designs and $\sqrt{s}$.

\begin{tabular}{|c|c|c|c|c|c|}
\hline $\mathrm{LC}$ & $\sqrt{s}(\mathrm{TeV})$ & $\mathrm{R}(\mathrm{cm})$ & Hits $\mathrm{mm}^{-2} \mathrm{BX}^{-1}$ & $25 \mathrm{~ns}^{-1}$ & $\operatorname{train}^{-1}$ \\
\hline NLC & 0.5 & 1.2 & 0.100 & 1.80 & 9.5 \\
\hline TESLA & 0.8 & 1.5 & 0.050 & 0.05 & 225.0 \\
\hline CLIC & 3.0 & 3.0 & 0.005 & 0.18 & 0.8 \\
\hline
\end{tabular}

While the pair background sets the most stringent constraint on the Vertex Tracker geometry, defining an inward bound on the detector radial position, track density in highly collimated hadronic jets contributes significantly to the local detector occupancy. Typical values for the local track densities are expected to be of the order of $0.2-1.0$ hit $\mathrm{mm}^{-2}$ at $\sqrt{s}=0.5 \mathrm{TeV}$, depending on the physics process, and about a factor three larger at $\sqrt{s}=3 \mathrm{TeV}$ for solenoidal fields of $4 \mathrm{~T}$ and $6 \mathrm{~T}$ respectively. Values for the anticipated total hit density are comparable to, or larger than, those foreseen on the innermost vertex detector layers at the LHC, ranging between $\simeq 0.03 \mathrm{hits} / 25 \mathrm{~ns}$ for ATLAS and 0.9 hits/25 ns in ALICE.

Other background sources may also be critical for the sensor design. Neutrons are copiously produced by the dump of pairs, beamstrahlung photons and spent beams. Their flux into the sensitive detector volume must be reduced by a proper choice of surface coatings and by isolating the detector from the accelerator tunnel. Detailed simulation of neutron production and transport 
show that the expected neutron fluxes are in the range of few times $10^{9} 1 \mathrm{MeV}$ equivalent neutrons $\mathrm{cm}^{-2}$ year $^{-1}$ for the NLC and TESLA and about an order of magnitude larger at a multi-TeV collider. While these fluxes are several orders of magnitude smaller compared to those expected at the LHC, they still impose special care for some of the proposed sensor technologies, such as Charged Coupled Devices (CCD), to minimise the inefficiencies arising from the creation of charge trapping sites due to bulk radiation damage. Finally $\gamma \gamma \rightarrow$ hadrons events overlayed on a $e^{+} e^{-}$interactions need to be minimised. In fact, the additional visible energy and the displaced particle vertex from $\gamma \gamma \rightarrow$ hadrons may distort the event reconstruction in channels with missing energy signatures and affect the jet flavour tagging performances (5). LC designs with large luminosity per bunch crossing or high $\sqrt{s}$ energy are more affected by the higher probability of a $\gamma \gamma$ event overlap. At $\sqrt{s}=350 \mathrm{GeV}$, this probability is $\simeq 0.02 \mathrm{BX}^{-1}$ and $\simeq 0.12 \mathrm{BX}^{-1}$ in the case of NLC and TESLA respectively and becomes $\simeq 4.0 \mathrm{BX}^{-1}$ at $\sqrt{s}=3 \mathrm{TeV}$ for the CLIC parameters. Fast time stamping, providing bunch identification information, will allow to reduce the total $\gamma \gamma$ overlap probability.

These issues emphasise the case for sensors with small pixel cells and fast detector read-out or fast time stamping capabilities. Setting an acceptable rate of background as 5 hits $\mathrm{mm}^{-2}$ and $<0.5 \gamma \gamma$ event for precision physics at $\sqrt{s}=350-500 \mathrm{GeV}$, results in the requirement to identify hits from events within $70 \mathrm{~ns}$ and $1 \mu \mathrm{s}$ for the NLC and TESLA projects. This requirement is well within the planned timing performances of the LHC pixel detector electronics and is being address by recent developments in CCD read-out (6).

\section{Physics Objectives for the Vertex Tracker}

The linear collider has the potential to cover the widest energy range for any particle accelerator operated so far, ranging from the $Z^{0}$ peak up to the multi-TeV region. Within this range, its physics programme covers precision electro-weak tests, a detailed study of the electro-weak symmetry breaking mechanism and the search for new physics at, and beyond, the energy scale probed by the LHC. This programme relies on efficient flavour identification to perform detailed studies of particle couplings to up- and down-type quarks and to different fermion generations, aiming to probe the Higgs mechanism of mass generation and new phenomena such as Supersymmetry and extradimensions, and to identify fermions of the heaviest generation $(\tau, b$ and $t)$ for searching for new particles, such as the heavy part of the Higgs sector in extensions of the Standard Model $\left(H^{ \pm}, H^{0}, A^{0}\right)$. Jet flavour tagging is based on the accurately measured track impact parameters, complemented by the topology of detected secondary and tertiary vertices and kinematical variables sensitive to the secondary track multiplicity and invariant mass of tau leptons and charm or beauty hadrons. 
In addressing this variety of processes, the anticipated S/B ratios and kinematical conditions vary quite considerably. At energies around the $Z^{0}$ peak the typical decay length in space of a $B$ hadron is $0.3 \mathrm{~cm}$ and about one in five $Z^{0}$ decays consists of a $b \bar{b}$ pair. The availability of data samples of the order of $10^{9}-10^{10} Z^{0}$ can improve the accuracy on $\Gamma_{b \bar{b}}$ and $A_{b}$ by factors of five and ten respectively, provided the $b$ tagging purity can be improved from $98 \%$, obtained by DELPHI, to $99.3 \%$ for a constant efficiency of $30 \%$ (7). The investigation of $\mathrm{CP}$ violation in the $B$ sector and of rare decays at the $B$-factory and $p p$ collider experiments, may be valuably complemented by studies profiting of the unique kinematical properties of $Z^{0} \rightarrow b \bar{b}$ decays.

At $\sqrt{s}=0.3-0.8 \mathrm{TeV}, b$-tagging is instrumental in isolating a clean Higgs signal, for Higgs masses up to about $150 \mathrm{GeV} / c^{2}$. The study of the Higgs boson (mass, width, quantum number and couplings) in the $e^{+} e^{-} \rightarrow H Z$ and $H \nu \bar{\nu}$ production processes needs a good rejection of the dominant $W W$ and $Z Z$ backgrounds, that can be achieved by selecting $H \rightarrow b \bar{b}$ decays with high efficiency. In the challenging Higgs-strahlung off top quark $t \bar{t} H$ and double Higgs production, $H H Z$ and $H H \nu \bar{\nu}$, reactions, backgrounds need to be rejected by almost five orders of magnitude in order to measure the top Higgs Yukawa coupling and the triple Higgs coupling, necessary to complete the investigation of the Higgs coupling properties. Four $b$-jets, in multi-jet final states from $t \bar{t} H \rightarrow b W^{+} \bar{b} W^{-} b \bar{b}, H H Z \rightarrow b \bar{b} b \bar{b} q \bar{q}$ and also Supersymmetric charged Higgs decays $H^{+} H^{-} \rightarrow t \bar{b} \bar{t} b \rightarrow b W^{+} \bar{b} \bar{b} W^{-} b$, offer a spectacular signature, while requiring high $b$-tagging efficiency due to the small production cross-section, corresponding to as few as 15 events per $100 \mathrm{fb}^{-1}$. At the same time, the large $H \rightarrow b \bar{b}$ yield poses a major challenge to the extraction of the tiny branching fractions for $H \rightarrow c \bar{c}$ and $H \rightarrow g g$ decays that are important as proof that the Higgs mechanism is responsible for the fermion mass generation and to identify the Standard Model or Supersymmetric nature of the neutral Higgs boson (8). This requires charm tagging performing efficiently in presence of both beauty and light quark backgrounds. This indicates the need of preserving an accurate track extrapolation resolution down to low momenta to tell the charm on the basis of the reconstructed mass and decay multiplicity of the detected short-lived hadron. Charm tagging is also instrumental in the study of triple gauge boson couplings that can be performed by analysing the reaction $e^{+} e^{-} \rightarrow W^{+} W^{-} \rightarrow \ell^{+} \nu q \bar{q}^{\prime}$ at high energy. The capability of tagging charm jets with $85 \%$ efficiency corresponds to a $50 \%$ equivalent luminosity gain compared to flavour blind detector results, where information is lost in the averaging of the $q \bar{q}^{\prime}$ states (9). $\tau$ leptons are expected to be a main signature for decay of Supersymmetric particles in models with large values of the $\tan \beta$ parameter. Their detection in jets requires to match the impact parameter tag with the calorimetric response.

While the developments of Vertex Trackers from the LEP and SLD experiments to those at B-factories, the Tevatron and the LHC have addressed many 
crucial aspects, such as radiation hardness, read-out speed and the considerable size increase of the trackers, the track extrapolation resolution, $\sigma_{i p}$, has not benefited of significant improvements. Limited in terms of multiple scattering the $B$ factories and by radiation damage in their closest approach to the beam the hadron colliders, their typical performances are comparable to those achieved at LEP, $\sigma_{i p}=25 \mu \mathrm{m} \oplus 70 \mu \mathrm{m} / p_{t} \mathrm{GeV} / c$. In order to fulfill its challenging requirements, the LC Vertex Tracker aims at significantly improving on the already outstanding SLD VXD3 performances of $8 \mu \mathrm{m} \oplus 33 \mu \mathrm{m} / p_{t}$ $\mathrm{GeV} / c$ (10). An improvement to $\sigma_{i p}$ to $5 \mu \mathrm{m} \oplus 10 \mu \mathrm{m} / p_{t} \mathrm{GeV} / c$ corresponds to a factor $\simeq 2$ increase in charm jet tagging efficiency and a factor $\simeq 1.25$ increase in beauty jet tagging efficiency at constant mis-identification probability. Since for a given detector geometry, $\sigma_{i p}$ depends on the sensor thickness and single point resolution, these requirements have motivated a dedicated R\&D activity to improve the pixel detector performances beyond what already achieved for the LHC experiments (11) and a study of a new concept of unsupported CCD sensors to minimise the material budget (6).

At multi-TeV energies, flavour dependent electro-weak observables, such as $\Gamma_{c \bar{c}}, \Gamma_{b \bar{b}}$ and $A_{f b}^{b \bar{b}}$, offer a window on new phenomena, such as new gauge bosons, compactified extra-dimensions and contact interactions, well beyond $10 \mathrm{TeV}$. The large $B$ hadron boost causes decay vertices to be located well beyond the first layers of the tracker and the decay products to be highly collimated. In $e^{+} e^{-} \rightarrow b \bar{b}$, the average $B$ decay length increases to $\simeq 10 \mathrm{~cm}$ with a local track density $>1 \mathrm{~mm}^{-2}$, thus requiring a large Vertex Tracker volume and, possibly, new jet flavour tagging methods extending the perspectives for developments of new generations of Vertex Trackers at $e^{+} e^{-}$colliders.

\section{References}

[1] T.O. Raubenheimer (editor), SLAC-Report-474 (1996).

[2] N. Toge (editor), KEK-Report 97-1 (1997).

[3] R. Brinkmann, G. Materlik, J. Rossbach and A. Wagner (editors), DESY 97-48 (1997).

[4] G. Guignard (editor), CERN-2000-008.

[5] S. Yamashita and J. Kanzaki, in Proc. of the Worldwide Study on Physics and Experiments with Future $e^{+} e^{-}$Linear Colliders, E. Fernandez and A. Pacheco (editors), UAB, Barcelona 2000, vol. I, 185.

[6] A. Gillman et al., these proceedings.

[7] R. Hawkings and K. Moenig, Eur. Phys. J. direct C8 (1999), 1.

[8] M. Battaglia, in Proc. of the Worldwide Study ..., cit., vol. I, 163.

[9] W. Walkowiak, to appear in Proc. of the Linear Collider Workshop LCWS 2000, Fermilab, October 2000.

[10] T. Abe for the SLD collaboration, Nucl. Instr. Meth. A447 (2000), 90.

[11] M. Battaglia et al., these proceedings. 